\title{
Multilinguales
}

\section{Quelques caractéristiques du discours procédural en tamazight : étude des consignes scolaires}

Some characteristics of the procedural speech in Tamazight : study of the school instructions

$$
\text { بعض خصائص الخطاب الإجرائي في الأمازيغية: دراسة التعليمات المدرسية }
$$

\section{Djohra Titouah et Zahir Meksem}

\section{OpenEdition}

\section{Journals}

\section{Édition électronique}

URL : http://journals.openedition.org/multilinguales/1136

DOI : 10.4000/multilinguales. 1136

ISSN : 2335-1853

\section{Éditeur}

Université Abderrahmane Mira - Bejaia

Référence électronique

Djohra Titouah et Zahir Meksem, «Quelques caractéristiques du discours procédural en tamazight étude des consignes scolaires », Multilinguales [En ligne], 9 | 2018, mis en ligne le 01 juin 2018, consulté le 17 septembre 2019. URL : http://journals.openedition.org/multilinguales/1136; DOI : 10.4000/multilinguales.1136

Ce document a été généré automatiquement le 17 septembre 2019.

\section{$\oplus \otimes \Theta$}

Multilinguales est mise à disposition selon les termes de la Licence Creative Commons Attribution Pas d'Utilisation Commerciale - Pas de Modification 4.0 International 


\title{
Quelques caractéristiques $\mathrm{du}$ discours procédural en tamazight : étude des consignes scolaires
}

\author{
Some characteristics of the procedural speech in Tamazight : study of the school \\ instructions \\ بعض خصائص الخطاب الإجرائي في الأمازيغية: دراسة التعليمات المدرسية
}

\author{
Djohra Titouah et Zahir Meksem
}

1 Le présent article fait partie de l'ensemble des études consacrées à la didactique de la langue amazighe. Nous nous intéresserons à l'étude de l'une des formes du discours procédural en langue amazighe, en l'occurrence la consigne scolaire. Notre propos consiste à faire une analyse des éléments qui constituent l'énoncé de la consigne dans le manuel scolaire de première année du collège de la langue amazighe. Nous avons opté pour l'analyse des spécificités linguistiques des consignes scolaires car, à notre connaissance, elles n'ont fait objet d'aucune étude scientifique. De plus, ce discours revêt une importance capitale puisqu'il est présent dans beaucoup de domaines de la vie sociale (les recettes de cuisines, les notices médicales, les guides touristiques, les conseils, les consignes de sécurité...). De même, en éducation, les consignes ont un rôle prépondérant quoique différent de celui qu'elles jouent dans la vie quotidienne. Elles sont considérées comme un moyen pédagogique que l'enseignant met à la disposition de l'élève pour assurer le contrat didactique. La question de la consigne dans ce milieu, qui est l'école, interpelle donc à chaque fois les enseignants.

2 La littérature scientifique consacrée à l'étude des consignes dans le contexte scolaire est abondante; et les chercheurs ont pris conscience qu'une bonne partie des difficultés rencontrées par les élèves dans la réalisation des exercices scolaires sont dues à l'incompréhension de l'énoncé de la consigne. C'est pourquoi il est nécessaire de prêter une très grande attention à sa formulation et, par conséquent, de s'interroger sur les éléments qui constituent l'énoncé de la consigne. 
3 L'étude des consignes en milieu scolaire peut être menée de deux manières différentes. La première se situe au niveau de la compréhension des consignes; elle concerne l'étude sémantique des représentations que se construisent les élèves au cours de la réalisation de l'exercice scolaire et les modalités cognitives qui interviennent lors de son exécution. La seconde concerne la formulation des consignes. Elle porte sur les éléments qui constituent ces énoncés. Elle s'intéresse alors à l'étude du vocabulaire et de la syntaxe.

4 Le présent article vise à mettre en exergue quelques spécificités intrinsèques de l'énoncé de la consigne. Il s'agit plus exactement de l'étude des formes linguistiques (vocabulaire et syntaxe) qui déterminent les consignes en langue amazighe dans le manuel scolaire de première année du collège.

5 Notre objectif à travers cet article consiste, dans un premier temps, à présenter les particularités linguistiques qui caractérisent le discours procédural, en langue amazighe, d'une manière générale, et des consignes scolaires d'une manière spécifique. Dans le second temps, notre intérêt consiste également à mettre en exergue quelques ambiguïtés qu'engendre parfois la formulation des consignes.

6 Nous essaierons dans le présent exposé de répondre à cet ensemble de questionnements :

Quelles sont les caractéristiques linguistiques des consignes dans le manuel scolaire de la première année de la langue amazighe?

Quels types de prédicats utilisés dans la formulation de ces consignes?

Quels sont les structures syntaxiques dominantes dans les énoncés de ces consignes?

7 Avant d'entamer cette étude, nous nous attacherons, après une brève présentation de notre matériau de travail (le corpus), à expliciter le sens de la consigne dans le milieu scolaire. Nous situerons également sa place dans la typologie textuelle. Ensuite, nous dégagerons les formes linguistiques qui caractérisent l'énoncé de la consigne.

\section{Le corpus d'étude}

8 Le corpus de notre étude est extrait du manuel de première année du collège. Le choix du corpus comme base d'analyse est motivé par l'étude que nous avons entamée dans le cadre de notre thèse sur les consignes dans les manuels scolaires du collège, dans laquelle nous avons constaté que les énoncés des consignes présentent des particularités linguistiques, à savoir la thématisation, l'ellipse et les prédicats actionnels.

9 Nous avons alors pris 14 consignes que nous avons soumises à une analyse linguistique. Pour chaque particularité syntaxique, nous avons choisi un ou deux exemples de consignes. Quant aux activités scolaires sur lesquelles nous avons travaillé, nous avons pris celles de la grammaire, de la conjugaison et de la notation. Ce sont les activités les plus intéressantes à l'étude, puisque le nombre de consignes est plus important. De plus, dans ces activités, nous remarquons que les consignes se manifestent par des formes syntaxiques plus variées.

10 Notre méthode d'analyse sera descriptive. Nous procéderons à une description des fonctions syntaxiques que peuvent avoir les unités significatives qui construisent les énoncés des consignes. Nous dégagerons aussi le sens de certaines unités pour montrer les difficultés de compréhension auxquelles l'apprenant peut faire face. 


\section{Le sens de la consigne dans le milieu scolaire} sapproprier, de maniere indispensable, elle est généralement classée dans le type injonctif. Selon Garcia-Debanc (2001:3), ce type a fait objet d'étude de plusieurs chercheurs dans divers champs disciplinaires qui lui ont donné des dénominations diverses (Pitar, 2015) : texte prescriptif, programmateur, texte procédural, textes de consignes, etc.

17 La revue Langages (2001) a même consacré un numéro spécial à l'étude de cette catégorie textuelle. L'intitulé du numéro est « discours procéduraux ». Selon Domingez Estefania,

les articles y sont rédigés par rapport à deux approches. La première approche a une orientation cognitive, elle s'intéresse au traitement cognitif de ces discours par les éventuels utilisateurs (saisie, compréhension et interprétation) (2013:11). typologie et de caractérisation des discours procéduraux. L'article de Jean-Michel Adam 
(2001) s'inscrit dans cette approche. L'auteur aborde les textes d'incitation à l'action par rapport à des régularités linguistiques.

Dans le présent article, nous focaliserons notre étude sur l'analyse des régularités linguistiques qui caractérisent les consignes scolaires.

\section{Analyse des données}

21 L'étude de la formulation des consignes peut être menée par une analyse des éléments inhérents à chaque énoncé. Pour pouvoir caractériser les consignes d'exercices dans la famille des textes procéduraux, nous nous intéressons, dans un premier temps, à l'étude des types de prédicat. Et à travers quelques exemples, nous mettons l'accent sur certaines caractéristiques linguistiques des consignes dans le domaine de la langue amazighe.

\section{Les types de prédicats dans les énoncés de la consigne}

Dans les énoncés de consigne, le verbe d'action est le noyau principal qui traduit un objectif en un comportement observable. En effet, il permet à l'élève de construire une représentation sur l'objectif à atteindre. Il est considéré comme un moyen qui oriente l'élève dans une situation de devoir ou d'examen en l'incitant directement à réaliser une tâche.

Les verbes utilisés, dans notre corpus, sont: smel (classer), bḍ (diviser), smed (compléter), err (mettre), kkes (enlever), af (trouver), efk (donner), semres (utiliser), seyti (corriger), sewnes (entourer). Le verbe le plus utilisé est le verbe af (trouver), il est répété dans 5 énoncés de consignes.

Chaque consigne contient un verbe d'action. Pour expliquer cela, nous prenons les exemples suivants :

Smed tafelwit-a « complète le tableau suivant »;

Err imyagen-a yer wurmir ussid « mets ces verbes à l'aoriste intensif »;

Kkes allus yellan deg tefyar-a s yimqimen iwsilen «évite les répétitions que

contiennent ces phrases en employant les pronoms affixes».

Comme nous pouvons le constater, le verbe d'action apparait en tête de l'énoncé dans les exemples sus-énoncés.

Le verbe peut également apparaitre au milieu de l'énoncé de la consigne quand il y a une anticipation ou un déplacement de la subordonnée. Les exemples suivants illustrent cela :

Yal arbib seg wigi, af-d arbib-nniden igan am netta deg talya «pour chacun des adjectifs suivants, trouve un autre adjectif qui a une même forme ";

Yal yiwen seg yimyagen-a, af-d talya taherfit-ines «pour chacun des verbes suivants, trouve la forme simple »;

Ha-ten-a yimyagen-a, smel-iten yef sin " voici les verbes, classe-les en deux ».

Dans notre corpus, nous avons trois exemples dans lesquels le verbe d'action est placé dans la deuxième partie de l'énoncé de la consigne.

Concernant le nombre d'exemples où le verbe d'action est placé en tête de l'énoncé, nous avons recensé 11 cas.

Comme première remarque, tous ces verbes ordonnent une action. En effet, ils incitent les élèves à agir en suivant les instructions de la consigne. 
Jean-Michel Adam a d'ailleurs constaté ce fait dans son étude sur des «textes qui disent de ou comment faire?» (2001:8) et que l'une des particularités de ces textes est la présence massive de prédicats actionnels. En effet, ces textes visent la transformation des comportements du destinataire, ici l'élève, en lui prescrivant un ensemble d'action à réaliser et en lui indiquant la manière de le faire.

\section{Les formes verbales et les marques d'énonciation qui se présentent dans les énoncés des consignes}

Dans la formulation d'une consigne, le verbe est un élément crucial car il permet de mettre directement l'élève en action. Dans l'énoncé de la consigne, les actions sont traduites par l'emploi de ces verbes à l'impératif. Cette forme verbale peut avoir plusieurs valeurs. En effet, nous utilisons l'impératif pour donner des conseils, des recommandations et même des ordres. L'impératif permet de rendre l'action à accomplir plus identifiable par les élèves.

Dans la langue amazighe, la forme la plus adéquate pour inciter à agir est également l'impératif. Ce mode verbal peut se présenter sous l'une des deux formes suivantes: l'impératif simple ou l'impératif intensif. Dans notre corpus, les verbes des consignes sont tous mis à la forme simple de l'impératif.

L'impératif simple se construit, en langue amazighe, à partir du schème d'aoriste (NaitZerrad, 2001: 85). Il ne s'emploie qu'avec deux personnes: la deuxième personne du singulier kečč, kemm (tu), et la deuxième personne du pluriel kunwi, kunemti (vous).

Concernant les déictiques personnels des verbes d'action que nous avons dans notre corpus : aru (écris), bdu (devise), sefti (conjugue), af (trouve), err (mets). Ils ne sont pas indiqués par une forme grammaticale. Chaker (2001:04) note à ce propos que

le paradigme particulier des indices de personnes de l'impératif manifeste très clairement son caractère «basique» voire archaïque : la $2^{\mathrm{e}}$ personne «tu » est caractérisée par un zéro morphologique, [...]

31 Nous comprenons que la forme qui marque l'indice de personne à l'impératif est représentée par la forme zéro ou ø, qui signifie l'absence d'une forme morphologique. Cette caractéristique concerne uniquement la $2^{\text {eme }}$ personne du singulier. Il en va autrement pour la deuxième personne du pluriel « vous » marquée par la présence de l'indice de personne-toum-dans arut/ arum "écrivez", seftit/ seftim "conjuguez » et bḍut/ bọum « divisez ». Ces désinences verbales sont toutes marquées par le morphème -t ou $\boldsymbol{m}$-qui indique le déictique personnel qui montre que l'énonciateur s'adresse à la deuxième personne du pluriel « vous ».

En somme, nous retenons que dans les verbes qui sont mis à l'impératif à la deuxième personne du singulier l'indice de personne n'est pas marqué par une forme grammaticale. Cela veut dire que c'est le contexte ou la situation d'énonciation qui détermine la personne à laquelle on s'adresse.

33 C'est d'ailleurs la référence au contexte de l'activité pédagogique (l'exercice en classe ou à la maison), mettant en relation l'élève (récepteur des consignes) et l'énonciateur (rédacteur des consignes) qui justifie l'usage du terme marques d'énonciation, dans le titre de ce paragraphe, à la place d'indices. 


\section{L'énonciateur dans une consigne écrite}

L'usage des déictiques personnels permet de reconnaitre directement la personne qui a émis la consigne. Dans une transmission orale des consignes, la présence de l'énonciateur est bien indiquée. Prenons l'exemple suivant :

Ad d-sutrey deg-wen ad iyi-d-tarum ${ }^{1} . .$. « je vous demande de me rédiger... »

Cet énoncé est un exemple d'une consigne orale que nous rencontrons souvent dans une interaction orale entre l'enseignant et les élèves. Dans cet énoncé, nous remarquons la présence d'un indice de personne " $\gamma$ » qui renvoie à celui qui a émis la consigne. Ce n'est cependant pas le cas dans une consigne écrite où on ne peut pas voir celui qui parle. Et même dans l'énoncé de la consigne écrite, on remarque qu'il n'ya pas de forme ou de marque grammaticale qui révèle la présence de celui qui parle ou de celui qui a émis cette consigne. Dans ce cas, l'énonciateur dans une consigne écrite est dans une situation différée.

Exemple: smel ismawen-a, bdu gar ukmam d umadwan² ${ }^{2}$ classe ces noms, fais une distinction entre le concret et l'abstrait ».

Dans cet exemple, nous remarquons qu'il n'ya pas de marque ou de forme grammaticale qui indique la présence de celui qui a énoncé la consigne. Ce constat est bien similaire à ce que Adam nomme "présence énonciative de l'énonciateur est effacée " (2001:22) dans les textes qui disent de/ comment faire.

En somme, nous pouvons dire que dans les textes qui disent de faire, les formes linguistiques renvoyant au sujet énonciateur sont souvent effacées.

37 A côté de toutes ces spécificités grammaticales et/ou énonciatives qui caractérisent l'énoncé de la consigne dans le contexte scolaire, nous pouvons aussi constater d'autres caractéristiques. Celles-ci relèvent de la forme grammaticale des énoncés de consigne. Dans ce qui suit, nous focaliserons notre analyse sur les particularités syntaxiques des énoncés de consignes de notre corpus.

\section{Les formes syntaxiques des consignes dans le manuel scolaire}

Dans le contexte scolaire les consignes se manifestent sous des aspects syntaxiques variés. Elles peuvent apparaitre sous la forme de phrases courtes et simples comme elles peuvent apparaitre sous la forme d'une phrase longue et complexe. Ces deux types de formes se trouvent très représentés dans notre corpus, renvoyant ainsi aux deux catégories de phrases : les phrases simples et les phrases complexes.

Dans notre corpus, nous avons trois formes distinctes : la première est la proposition coordonnée, la seconde est la proposition subordonnée relative et la troisième et la proposition subordonnée conjonctive.

\section{La forme simple/ phrase simple}

Nous avons recensé 5 consignes qui se présentent sous forme de phrase simple. Pour présenter les éléments qui constituent cette forme de phrase, nous prenons les exemples suivants. 


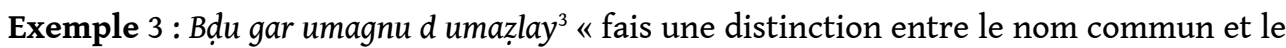
nom propre ".

Dans le verbe $b d u$ « diviser, distinguer » l'indice de personne n'est pas marqué [(forme $ø$ : + verbe (impératif) + préposition (gar+ $\mathrm{COI}_{1}($ umagnu $)+$ préposition $(d)+\mathrm{COI}_{2}$ (amazlay)].

41 Cette phrase est constituée d'un verbe qui est conjugué à l'impératif ; elle se compose de deux prépositions et deux compliments d'objet indirects. Nous la considérons comme une phrase simple car elle ne contient qu'un seul prédicat verbal bḍ « divise, distingue ». En plus, cette consigne est simple car elle demande à l'élève d'effectuer une seule tâche voire une seule action.

Exemple 4 : Err imyagen-a yer wurmir ussid ${ }^{4}$ " mets ces verbes à l'aoriste intensif » Cette phrase est de même catégorie ; elle se compose d'un verbe qui est conjugué au mode impératif ; l'indice de personne dans ce verbe n'est pas marqué. A côté du verbe, nous trouverons le COD = imyagen- $a+$ préposition " yer » + COI « wurmir ussid ".

Cette analyse des deux énoncés des consignes rentre dans la sphère de l'analyse syntaxique. Car nous avons essayé de dégager les fonctions des unités qui constituent l'énoncé de la consigne.

C'est à partir de ces deux formes (présence d'un seul prédicat verbal), que nous pourrions dire que les consignes dans le milieu scolaire peuvent apparaitre sous forme de phrases simples.

\section{Les formes composées ou complexes}

Nous avons recensé 9 consignes qui se manifestent sous forme de phrases complexes. Celles-ci sont constituées de deux prédicats ou plus, et donc de deux propositions ou plus. Si dans les exemples traités ci-dessus, le prédicat est toujours verbal, l'exemple qui suit fera l'exception : le prédicat est un syntagme nominal ou non verbal. C'est ce qu'illustre la consigne $n^{\circ} 5$ de notre corpus.

Exemple 5 : Ha-ten- $a^{5}$ yimyagen, smel-iten $\gamma e f \sin ^{6}$ « voici ces verbes, classe-les en deux catégories "

En tamazight $\mathrm{Ha}$ « voici » appartient à la catégorie des pronoms démonstratifs. Ce qu'il faut noter dans cet exemple est que cette consigne a la forme d'une phrase composée avec deux prédicats : le premier, ha-ten-a, est un syntagme prédicatif nominal, non actionnel, donc non verbal, et le second l'est, puisqu'il commence par un verbe smel « classe ».

Ce qu'il faut retenir dans l'analyse de cet exemple, c'est que dans les cas où il y a une thématisation, la partie informative d'une consigne est introduite par des prédicats non verbaux. Ces derniers peuvent rendre la demande de faire plus attrayante. En d'autres termes, dans un énoncé de la consigne, la partie injonctive est indiquée par un verbe ou par un syntagme prédicatif actionnel et la partie informative est représentée par un syntagme prédicatif non verbal.

46 Revenons maintenant à la phrase composée avec la présence de deux syntagmes prédicatifs verbaux.

Exemple 6: Smel ismawen-a, bọu gar ukmam d umadwan ${ }^{7}$ "classe ces noms, distingue le nom concret du nom abstrait ».

Dans cet exemple, nous constatons qu'il ya présence de deux syntagmes prédicatifs verbaux, le premier est smel, le deuxième est bdu. Sur le plan syntaxique, nous pouvons 
remarquer que l'élève doit accomplir deux tâches puisqu'il y a présence de deux verbes d'action.

Cependant, sur le plan sémantique, ces verbes ne renvoient qu'à une seule tâche que l'enseignant a préféré diviser en deux pour faciliter son exécution par l'élève. Ce dernier ne pourra pas faire une classification des noms sans avoir réalisé au préalable une distinction entre eux.

Ce qu'il faut retenir de l'analyse de cet exemple est que la consigne complexe n'est pas toujours celle qui contient deux verbes d'action. C'est, en effet, à travers le sens des verbes que nous pouvons savoir si la consigne demande à l'élève d'exécuter deux tâches différentes ou non. En somme, le sens reste l'élément crucial qui détermine l'appartenance typologique des consignes.

Exemple 7: af-d tizelyiwin $n$ tnila ma llant, sewnes-itent ${ }^{8}$ " trouve les particules de direction, si elles existent,cerne-les ".

Dans cet exemple, nous avons aussi deux syntagmes prédicatifs verbaux : af et sewnes. Ces verbes représentent deux demandes d'action distinctes. L'exécution de la deuxième tâche dépend de la première tâche. En d'autres termes, dans cette structure complexe, la première proposition af-d tizelyiwin n tnila " trouve les particules de direction " constitue la tâche principale que l'élève est censé réaliser. A cette injonction, s'ajoute une autre demande qui est sewnes-iten »cerne-les ». L'exécution de cette deuxième tâche dépend de la première tâche. En effet, dans ce type de consignes l'élève ne pourra pas procéder à la réalisation de la deuxième tâche sans avoir réalisé la première demande.

50 La comparaison des deux exemples ci-dessus nous révèle une information importante : la tâche principale demandée pour exécution par l'élève est contenue dans la deuxième proposition de la phrase complexe ou de la consigne.

\section{La proposition coordonnée par juxtaposition}

51 Dans notre corpus, nous avons recensé 4 exemples de phrases coordonnées par juxtaposition. Nous avons recours à ce type de consignes quand les objectifs qui sont fixés dans les exercices ne se réalisent pas par une simple demande de faire et/ou pour ordonner les différentes étapes de la réalisation d'une tâche. D'où la nécessité de présenter à chaque fois deux consignes dans un seul exercice. La proposition coordonnée consiste donc à une suite de deux propositions verbales ou plus. L'exemple suivant en témoigne de cette caractéristique : smel ismawen-a, bdu gar ukmam d umadwan " classe ces noms, distingue le nom concret du nom abstrait » Smel ismawen-a structure verbale 1, Bḍ gar ukmam d umadwan structure verbale 2 .

52 Entre la structure verbale 1 et la structure verbale 2, nous constatons qu'il n'ya pas de conjonction de coordination. En effet, les deux propositions sont juxtaposées. Sur le plan syntaxique, elles sont indépendantes. Si à l'oral, c'est la pause qui marque la juxtaposition, à l'écrit, elle est signalée par la virgule.

\section{La proposition subordonnée}

Dans notre corpus, nous avons constaté la présence de deux types de subordonnées. La première est la proposition subordonnée introduite par une conjonction de subordination. Quant à la deuxième, c'est la proposition subordonnée relative. 


\section{La proposition subordonnée conjonctive} les lignes qui suivent.

\section{Les énoncés elliptiques}

61 On parle d'ellipse quand il y a l'absence d'un mot ou de plusieurs éléments dans la phrase et sans que cela modifie son sens. Selon Dubois (2012:174): «On qualifie 
d'elliptiques certaines phrases incomplètes, inachevées dans lesquelles il manque un élément structurel». Nous notons l'existence de cette caractéristique syntaxique dans 2 exemples de notre corpus.

Exemple 10 : Efk-d snat $n$ tefyar ad yili deg-sent yisem amalay, snat-nniden ad yili deg-sent isem unti ${ }^{11}$ " Donne deux phrases avec un nom masculin et deux autres avec un nom féminin ».

Nous remarquons dans cet exemple qu'il y a l'omission du verbe d'action dans la deuxième partie de l'énoncé. La structure de la consigne sans l'ellipse est : efk-d snat $n$ tefyar ad yili deg-sent yisem amalay, (efk-d) snat-nniden ad yili deg-sent yisem unti. Il y a une omission du verbe d'action pour faire l'économie de la langue en évitant la répétition du verbe d'action qui est déjà présent en tête de l'énoncé de la consigne.

Exemple 11 : Semres imerna deg tefyar (ara d-tefked) syur- $k / \mathrm{m}^{12}$ (utilise des adverbes dans des phrases (que tu formuleras) toi-même ».

Dans cet exemple, l'élément omis est " ara d-tefkeḍ », cette structure elliptique dans ce cas est due, à notre sens, à une formulation inadéquate de la consigne.

63 A partir de ces deux exemples, nous pouvons dire que la tournure elliptique ou l'absence d'un autre verbe d'action peut altérer le sens de la consigne. Cela pourrait même engendrer une difficulté dans la compréhension de la tâche demandée. Ce constat a été fait au cours d'une enquête réalisée dans le cadre de notre travail de thèse de doctorat. En effet, certains élèves ont du mal à saisir ce que cet énoncé leur est demandé de faire "Semres imerna deg tefyar (ara $d$-tefked) syur-k/m», la question que se posaient les élèves est la suivante : " qu'est-ce que veut dire le syntagme sður-k/m?». Ils ne savent donc pas qu'il faut d'abord donner des phrases. Ce problème d'incompréhension est dû, à notre sens, à l'omission du syntagme verbe ara d-tefked introduit par le relatif $i$ amalgamé (i ad d-tefkeḍ).

64 Une autre possibilité est envisageable en reformulant la consigne de telle sorte d'avoir deux propositions liées par un tout autre relatif ou conjonction comme anda " où » : efk$d$ tifyar anda ara tsexdmed imerna "donne des phrases où tu introduis des adverbes". Ainsi, nous pouvons dire que les énoncés elliptiques peuvent être à l'origine d'une mauvaise compréhension des tâches scolaires.

\section{La thématisation}

C'est une caractéristique syntaxique qui est souvent présente dans les énoncés des consignes dans le manuel scolaire. La thématisation est le fait d'introduire un énoncé par un syntagme nominal à l'état libre. Cette caractéristique syntaxique est représentée dans 3 exemples dans notre corpus. Selon Achour Remdane (2017: 239): «la thématisation sert à mettre en valeur des éléments importants dans une phrase et rendre la visée de la communication plus forte».

Exemple 12: Yal yiwen seg yimyagen-a, af-d talya tahefit-ines ${ }^{13}$ « chacun de ces verbes, trouve (donne) sa forme simple».

Dans cet énoncé, la thématisation se situe avant le syntagme prédicatif verbal "af». L'indicateur du thème dans ce cas c'est "yal yiwen seg yimyagen-a ». L'énoncé anticipé est constitué de : nom+préposition+nom. 
L'énoncé neutre de cette consigne est : af-d talya taherfit $n$ yal yiwen seg yimyagen » trouve la forme simple de chacun de ces verbes), dans cet énoncé, le syntagmeyal yiwen seg yimyagen-a est un complément du nom.

Les deux énoncés: "Yal yiwen seg yimyagen-a, af-d talya tahefit-ines " et "af-d talya taherfit n yal yiwen seg yimyagen " sont identiques sur le plan syntaxique, il y a seulement une anticipation du complément de nom.

Nous rencontrons souvent cet aspect syntaxique dans des sujets d'examens et/ou des devoirs scolaires, dans les consignes d'une manière générale. La thématisation dans ce cas a pour objectif de donner plus d'informations concernant la tâche à réaliser. Mais dans certaines situations, nous présumons que ces tournures syntaxiques peuvent créer une certaine confusion dans l'esprit de l'élève puisque la consigne a pour objectif premier de dire ce qu'il faut faire ou comment faire. Elles devraient, par conséquent, être introduites par un verbe qui demande une action.

En somme, nous pouvons dire que l'emplacement du verbe dans l'énoncé de la consigne a un rôle capital, car dans certaines consignes, le verbe n'intervient qu'à la fin de l'énoncé et cela peut, en effet, distraire l'attention de l'élève.

68 En guise de conclusion, nous avons dégagé, à travers cet article, certains aspects linguistiques que nous considérons comme des caractéristiques des consignes scolaires. Dans un premier temps, nous avons inscrit les consignes scolaires dans le cercle des textes qui disent de faire/ textes procéduraux, en nous référant aux régularités linguistiques qu'Adam a pu dégager à partir d'une étude sur les corpus des textes qui disent de ou comment faire.

69 Nous avons également constaté que les verbes d'action dans les consignes du manuel scolaire de $1^{\text {ere }}$ année sont alors conjugués au mode de l'impératif simple et à la deuxième personne du singulier cela est dû à l'inexistence du vouvoiement dans la langue amazighe. Alors qu'en français c'est la deuxième personne du pluriel qui est la plus usitée. A vrai dire, quand on dit "transformez, rédigez, commentez", etc., il est difficile de savoir si l'on s'adresse au groupe-classe ou s'il s'agit du vouvoiement.

70 Notre analyse nous a permis d'avancer que les consignes apparaissent sous des formes syntaxiques diverses, à savoir, énoncé simple, complexe, énoncé elliptique, énoncé avec thématisation.

71 Cette étude n'est évidemment pas exhaustive. Certains aspects ne sont pas pris en compte, comme par exemple l'usage des prépositions, des affixes, les questions de détermination et la complexité des énoncés de consigne. A côté de ces préoccupations d'ordre linguistique, formelles et sémantiques, d'autres à dimension cognitive ou psycholinguistique liées à la saisie, à la compréhension et/ou à l'interprétation des consignes par les utilisateurs (élèves) sont également envisageables. Ce sont donc autant de perspectives ouvertes et de terrains qui attendent d'être analysés. 


\section{BIBLIOGRAPHIE}

ACHOUR, R., "structures phrastiques et fonctions syntaxiques en Kabyle », thèse de Doctorat, université de Mouloud Mammeri Tizi Ouzou, 2017.

ADAM, J-M., » Entre conseil et consigne : les genres de l'incitation à l'action », Pratiques, $\mathrm{N}^{\circ} 111-112$, 2001.

ADAM, J-M., «Types de textes ou genres de discours ? Comment classer les textes qui disent de et comment faire? », Langages, $\mathrm{N}^{\circ} 141,2001$, pp. 10-27.

RIEUNIER, A., et RAYNAL, F, Pédagogie : Dictionnaire des concepts clés, ESFEditeur, Paris, 1997.

CHAKER, S., « Impératif », in Gabriel Camps (dir.), 24 | Ida - Issamadanen, édition Edisud, Aix-en-

Provence, 2001.

CHAKER, S., « Fonctions », in Gabriel Camps (dir.), 19 | Filage - Gastel, Aix-en-Provence, 1998.

DUBOIS, et al., Dictionnaire de linguistique et des sciences du langage, édition Larousse, Italie.2012.

DOMINGUEZ, E., L'enseignement du FLE et l'activité de prescription : modalité (pré-réfléchit), Mémoire de Master, 2012-2013.

FAYOL, M., GOMBERT, J-É., GANIER, F., « Discours procédural et activités mentales : de la compréhension d'instructions complexes à la planification de l'action », Langages, $\mathrm{N}^{\circ} 141,2001$, pp. 47-63.

GARCIA-DEBANC, C., » étude des discours procéduraux aujourd'hui : travaux linguistiques et psycholinguistiques ", Langages, $\mathrm{N}^{\circ} 141,2001$.

MINISTERE DE L'EDUCATION NATIONALE, Adlis-w $n$ tmazi $t$ : aseggas 1 ru $n$ Ulemmud Alemmas, ONPS, Alger, 2013-2014.

NAIT ZERRAD, K., Grammaire moderne du kabyle », Edition Karthala, Paris, 2001.

RIVIÈRE, V., « Dire de faire, consigne, prescriptions .... Usages en classe de langue étrangère et seconde », Le français dans le monde, recherches et applications, 2008.

\section{NOTES}

1. Cet exemple n'est pas représenté dans notre corpus.

2. Cette consigne correspond au numéro 1 dans notre corpus.

3. La consigne correspond au numéro 2 dans notre corpus.

4. C'est la troisième consigne de notre corpus.

5. Nous n'avons pas gardé le figement des présentatifs car c'est de cette manière qu'ils se présentent dans le manuel scolaire.

6. La consigne correspond au numéro 5 dans notre corpus.

7. C'est la première consigne de notre corpus.

8. La consigne correspond au numéro 12 dans notre corpus.

9. La consigne correspond au numéro 10 dans notre corpus.

10. La consigne correspond au numéro 11 dans notre corpus.

11. La consigne correspond au numéro 8 dans notre corpus.

12. La consigne correspond au numéro 9 dans notre corpus. 


\section{RÉSUMÉS}

Le présent article fait partie de l'ensemble des études consacrées à la didactique de la langue amazighe. Il s'agit plus exactement de faire une analyse linguistique des consignes dans le contexte scolaire. Nous avons opté pour l'étude de ce discours car, à notre connaissance, il n'a fait l'objet d'aucune étude scientifique en langue amazighe. Notre objectif est de démontrer les spécificités linguistiques qui caractérisent les consignes scolaires en nous interrogeant sur la manière avec laquelle les concepteurs des manuels formulent les énoncés des consignes. Nous avons entrepris une analyse des prédicats verbaux, des déictiques personnels qui marquent l'ancrage énonciatif de la consigne écrite, ainsi que les diverses tournures syntaxiques qui sont présentes dans les énoncés des consignes.

This article is part of a set of studies devoted to didactics of the Amazigh language. It is more accurately a linguistic analysis of instructions statements in a school context. We have chosen to study this discourse because, to our knowledge, it has not been subject of any scientific study. Our goal is to show the linguistic specificities that characterize school instructions by questioning the way in which the designers of manuals formulate the statements of instructions. We have undertaken an analysis of verbal predicates, personal deictics that mark the enunciative anchoring of written instruction, and the various syntactic forms that are present in the statements of instructions.

\section{INDEX}

Mots-clés : consigne scolaire, discours procédural, marque d'énonciation, prédicat verbal, langue amazighe

$$
\text { التعليمات المدرسية, الخطاب الإجرائي, علامات الكلام, الألفاظفهرس الكلمات المفتاحية: }
$$

Keywords : school instructions, procedural speech, mark of enunciation, verbal predicates, amazigh language

\section{AUTEURS}

\section{ZAHIR MEKSEM}

Laboratoire LAILEMM Université Bejaia 